\title{
ON THE DEFINITION AND PROPERTIES OF CERTAIN VARIATIONAL INTEGRALS
}

\author{
BY \\ JAMES SERRIN
}

In this paper we shall be concerned with certain multiple integrals which arise in the calculus of variations, namely those of the form

$$
I[u]=\int_{R} f\left(x, u, u_{x}\right) d x .
$$

Here $R$ denotes an open set in the number space $E^{n}$ and $u=u(x)$ is a realvalued function defined on $R$. The integrand $f(x, u, p)$ is assumed to be nonnegative and continuous. Moreover, we suppose throughout the paper that $f$ is convex in $p$ so that $I[u]$ is the integral of a regular variational problem.

For the purposes of the calculus of variations, and also for aesthetic reasons, it is natural to want the class of admissible functions $u$ to be as large as possible. Now $I[u]$, as it stands, is certainly well-defined for continuously differentiable functions, but once we go beyond this class there is some question as to the meaning of the integral. If measurable partial derivatives can be associated with $u$, then one can define $I[u]$ simply as the Lebesgue integral of $f\left(x, u, u_{x}\right)$. This procedure cannot be used indiscriminately, however, for it assigns the absurd value $\int f(x, u, 0) d x$ to any nonconstant function $u$ whose partial derivatives are zero almost everywhere. As an alternate definition of the integral, we have introduced in [13] a certain lower semicontinuous functional which in general agrees with $I[u]$ whenever $u$ is continuously differentiable, but which at the same time is defined for a much larger class of functions. For convenience in discussing these two integrals the former will be denoted simply by $I[u]$ and the latter by $g[u]$, (a formal definition of these quantities will be given in $\S 1$ ). Both functionals $I[u]$ and $g[u]$ are of interest in the calculus of variations; it is the purpose of this paper to clarify the relation between them.

An important illustration of the present situation may be found in the theory of area of a nonparametric surface. Indeed, let us denote by $a[u]$ the Lebesgue area of a surface $z=u(x, y)$ over a region $R$ in the ordinary $(x, y)$ plane, and set

$$
A[u]=\int_{R}\left(1+u_{x}^{2}+u_{y}^{2}\right)^{1 / 2} d x d y .
$$

The functional $a[u]$ then stands in essentially the same relation to $A[u]$ as

Received by the editors March 10, 1961. 
$g[u]$ does to $I[u]$. Because of this, the theory of surface area can be used as a guide to the type of results which can be expected in the present more general situation. For example, it is known that $a[u]=A[u]$ whenever $u$ has strong derivatives, but that equality does not hold beyond this class of functions. A corresponding result for the functionals $I[u]$ and $g[u]$ will be one of the goals of this paper.

The paper is divided into three parts. The first part contains, in addition to definitions and other preliminary material, a discussion of the special case when the integrand depends only on $p$. The results here are especially simple and elegant, and serve as motivation for much of the later work. $\$ \$ 2$ and 4 contain generalizations of the theorem of Tonelli noted in the preceding paragraph. Because of a consistent use of integral averaging the proofs are actually simpler than the corresponding ones given in the theory of surface area (cf., for example, [11, Chapter V]). In $\$ 5$ we show that $g[u]$ is a convex functional of $u$, in analogy with the so-called Steiner inequality for surface area.

The results of Part I are extended to a significantly larger class of integrands in Part II. For a detailed idea of the results of Part II the reader is best referred directly to the theorems there. Theorem 10 , in particular, deserves special notice as our ultimate generalization of Tonelli's theorem.

There are a number of integrands which do not satisfy the conditions imposed in Parts I and II, but which nevertheless are amenable to an alternate approach. In Part III we investigate the functionals $I[u]$ and $g[u]$ corresponding to these more difficult integrands. Although the results are no longer so definitive, the greater generality of the integrands lends its own interest. The major effort turns on proving certain lower semicontinuity theorems for the integral $I[u]$, these being used to show, in particular, that $I[u] \leqq g[u]$ for the corresponding integrands. Since these lower semicontinuity theorems have an independent interest, some of them may be separately noted here. For example, Theorem 11 shows that $I[u]$ is lower semicontinuous with respect to weak convergence in $L_{1}$ whenever $f$ depends on $p$ alone, or even is of the form $A(x) F(p)$. In Theorem 12 we show that $I[u]$ is lower semicontinuous with respect to (strong) convergence in $L_{1}$ if $f$ is strictly convex, or if the derivatives $f_{x}, f_{p}$, and $f_{p x}$ exist and are continuous, or finally if only $f \rightarrow \infty$ as $|p| \rightarrow \infty$. These results, which are probably known for the case of one independent variable, generalize well-known multiple integral semicontinuity theorems of Tonelli and Morrey.

\section{PART I}

1. Definitions and preliminary results. Consider a real valued function $\phi=\phi(x)$ defined on an open set $R$ in the $n$-dimensional number space $E^{n}$. The function $\phi$ is called locally summable if it admits a finite Lebesgue integral over any compact subset of $R$. It is natural to associate a special kind of con- 
vergence with locally summable functions; namely, a sequence $\left\{\phi_{m}\right\}$ will be said to converge locally to $\phi$ in $R$ if for any compact subset $S$ of $R$ the following condition is satisfied:

$\phi_{m}$ is defined and summable in $S$, at least for all $m$ sufficiently large, and $\int_{s}\left|\phi_{m}-\phi\right| d x \rightarrow 0$ as $m \rightarrow \infty$.

To indicate that a sequence $\left\{\phi_{m}\right\}$ converges locally to $\phi$ we shall write $\phi_{m} \rightarrow \phi$.

The function $\phi$ is called strongly differentiable in $R$ if there exists a vectorvalued function $\psi$ such that

$$
\int \phi \omega_{x} d x=-\int \omega \psi d x
$$

for any continuously differentiable function $\omega$ with compact support in $R$. It $\phi$ is strongly differentiable there exists a sequence $\left\{\phi_{m}\right\}$ of continuously differentiable functions such that

$$
\phi_{m} \rightarrow \phi, \quad \phi_{m x} \rightarrow \psi .
$$

This condition is, in fact, often used to define strong differentiability.

In analogy with the concept of strong differentiation, we say that $\phi$ is weakly differentiable in $R$ if there exists a vector-valued Borel measure $\alpha$ such that

$$
\int \phi \omega_{x} d x=-\int \omega d \alpha
$$

for any continuously differentiable function $\omega$ with compact support in $R$. In general $\alpha$ need not be defined on the set $R$ itself; however, for the results of Theorems 3, 5, and 10 it is convenient to assume that $R$ is measurable and that $\alpha(R)$ is finite. It is evident that the class of weakly differentiable functions includes all strongly differentiable functions. In particular, by virtue of the Radon-Nikodym theorem a weakly differentiable function is strongly differentiable if and only if its derivative measure $\alpha$ is absolutely continuous. In this case the relation between $\alpha$ and $\psi$ is simply $\alpha(E)=\int_{E} \psi d x$.

If $\phi$ is weakly differentiable, its derivative measure is an additive set function. Consequently, we may define the derivative $\phi_{x}$ of $\phi$ by the formula

$$
\phi_{x}=\alpha^{\prime},
$$

where $\alpha^{\prime}$ denotes the general derivative( $\left.{ }^{1}\right)$ of $\alpha$. The function $\phi_{x}$ is then welldefined, equals $\psi$ when $\phi$ is strongly differentiable, and finally agrees with the

( ${ }^{1}$ Cf. $\left[11\right.$, pp. 106, 115]. Equivalently, $\alpha^{\prime}$ can be defined as the Radon-Nikodym derivative of the absolutely continuous part of $\alpha$, cf. [4, p. 133]. In either case, $\alpha$ and $\alpha^{\prime}$ are connected by the fundamental relation

$$
\alpha(E)=\int_{E} \alpha^{\prime}(x) d x+\theta(E),
$$

where $\theta(E)$ is a singular measure. 
ordinary partial derivatives of $\phi$ when $\phi$ is continuously differentiable. A fuller discussion of the properties of weakly differentiable functions will be found in references [12] and [14].

Integral averages. In certain of our results the operation of integral averaging or smoothing can be used to particular advantage. We associate with each positive number $h$ a corresponding function $K(\xi)=K(\xi, h)$ with the properties

(i) $K$ is non-negative and smooth for all values of $\xi=\left(\xi_{1}, \cdots, \xi_{n}\right)$.

(ii) $K \equiv 0$ for $|\xi| \geqq h$.

(iii) $\int K(\xi) d \xi=1$.

The $\xi$ integration in this and subsequent formulas is assumed to be carried out over the entire $n$-dimensional number space. Now let $\phi$ be a locally summable function in an open region $R$, and let $R_{h}$ denote the subset of $R$ whose points are farther than $h$ from the boundary of $R$. The integral average $\phi_{h}$ of $\phi$ is then defined by the formula

$$
\phi_{h}=\phi_{h}(x)=\int K(\xi-x) \phi(\xi) d \xi, \quad x \in R_{h} .
$$

It is well known and easy to show that $\phi_{h} \rightarrow \phi$ as $h \rightarrow 0$. Furthermore, $\phi_{h}$ is continuously differentiable and its derivative is given by the formula

$$
\phi_{h x}=-\int K_{\xi}(\xi-x) \phi(\xi) d \xi .
$$

From (1) and (6) it follows that if $\phi$ is strongly differentiable, then

$$
\phi_{h x}=\phi_{x h} .
$$

It is this equation, in fact, which justifies the relations (2); we need only choose $\phi_{m}=\phi_{h}$ with $h=m^{-1}$.

The functionals $I[u, R]$ and $g[u, R]$. The integrands $f(x, u, p)$ which we shall consider in this paper are assumed to be continuous for all $x$ in some open set $R_{0}$ and for all values of $u$ and $p$. In addition, without further mention we assume that

$$
f \geqq 0, \quad f \text { convex in } p .
$$

Now let $R$ be an open set, $R \subseteq R_{0}$, and let $u$ be a weakly differentiable function in $R$. We define

$$
I[u, R]=\int_{R} f\left(x, u, u_{x}\right) d x,
$$

the integral being understood in the sense of Lebesgue. Since $f$ is non-negative, the value $+\infty$ is allowed for $I[u, R]$. We shall occasionally write $I[u]$ instead of $I[u, R]$, provided the meaning is clear from the context. 
To define the functional $g[u, R]$ we proceed somewhat analogously to Lebesgue's definition of surface area. Let $R$ be an open set, $R \subseteq R_{0}$, and let $u$ be a locally summable function defined on $R$. Consider a sequence $\left\{u_{m}\right\}$ of continuously differentiable functions such that $u_{m} \rightarrow u$ in $R$. We set

$$
A=\lim \inf I\left[u_{m}, R_{m}\right],
$$

where $R_{m}$ denotes the (open) domain of definition of $u_{m}$. Then $\delta[u, R]$ is defined as the infimum of the numbers $A$ corresponding to all possible sequences $\left\{u_{m}\right\}$ converging to $u$ as above. By a diagonal process it is evident that there exists some sequence $\left\{u_{m}^{\prime}\right\}$ such that $\delta[u, R]=\lim I\left[u_{m}^{\prime}, R_{m}^{\prime}\right]$. Hence we can express the above definition in the concise form

$$
g[u, R] \equiv \underset{u_{m} \rightarrow u}{\operatorname{Minimum}} \operatorname{limit} I\left[u_{m}, R_{m}\right]
$$

It is an immediate consequence of this definition that $g[u, R]$ is lower semicontinuous with respect to local convergence. Also, let $u$ be a fixed function defined in $R \subseteq R_{0}$, and let $Q$ be a variable open subset of $R$. Then $g[u, Q]$ is a well-defined function of the set $Q$. For later use we observe that this set function is both monotone and countably additive, and in particular

$$
g\left[u, Q_{1}\right]+g\left[u, Q_{2}\right] \leqq g[u, R]
$$

if $Q_{1}$ and $Q_{2}$ are disjoint open subsets of $R$.

Certain slightly different definitions might be advocated for the functional $g[u, R]$. Some of these are discussed in $\$ 13$, to which the reader is referred. Another would replace local convergence by uniform convergence, in exact analogy with the procedure for Lebesgue area. It can be shown that the present definition is an extension of the latter one, in that both give the same value for continuous functions $u$ while only the present definition applies at all to discontinuous functions (see reference [13]).

2. The integrand $f=f(p)$. For the rest of Part I we shall be concerned with the properties and relationships of the functionals $I[u, R]$ and $g[u, R]$ when the integrand depends only on $p$. In the following lemma, and throughout the rest of the paper, the region $R_{m}$ denotes the (open) domain of definition of a function $u_{m}$. On the other hand, $R_{h}$ is the set of points of $R$ whose distance from the-boundary is greater than $h$.

LEMMA 1. Let $u$ be a locally summable function defined on an open set $R$, and let $\left\{u_{m}\right\}$ be a sequence of strongly differentiable functions converging locally to $u$ in $R$. Then for each $h>0$, we have

$$
I\left[u_{h}, R_{h}\right] \leqq \lim \inf I\left[u_{m}, R_{m}\right] .
$$

Proof. Let $S$ denote a fixed compact subset of $R$. Since $u_{m} \rightarrow u$ in $R$, it is clear that the domains $R_{m}$ eventually contain $S$, say for all $m \geqq m_{0}$. By virtue of (6) we have then for all $x \in S_{h}$ and $m \geqq m_{0}$, 
Therefore

$$
\left|u_{m h s}-u_{h s}\right| \leqq C(h) \int_{s}\left|u_{m}-u\right| d x .
$$

$$
f\left(u_{h x}\right) \leqq f\left(u_{m h x}\right)+\epsilon=f\left(u_{m x h}\right)+\epsilon \leqq f\left(u_{m x}\right)_{h}+\epsilon
$$

by Jensen's inequality, where $\epsilon=\epsilon(m, h)$ tends to zero as $m \rightarrow \infty$. Integrating this inequality over $S_{h}$ and applying Fubini's theorem yields

$$
I\left[u_{h}, S_{h}\right] \leqq \int_{S_{h}} f\left(u_{m x}\right)_{h} d x+\in \operatorname{Meas} S \leqq \int_{S} f\left(u_{m x}\right) d x+\in \operatorname{Meas} S ;
$$

in this transformation we have made essential use of properties (i), (ii) and (iii) of the kernel $K$ and the fact that $f \geqq 0$. Since $S \subset R_{m}$ the preceding inequality may be written

$$
I\left[u_{h}, S_{h}\right] \leqq I\left[u_{m}, R_{m}\right]+\in \text { Meas } S .
$$

Now let $m \rightarrow \infty$ and then $S \rightarrow R$, and the lemma is proved.

The following two theorems are simple consequences of Lemma 1 . The first gives a constructive procedure for evaluating $\mathscr{g}[u, R]$, analogous to Radó's theorem for the evaluation of Lebesgue area (see reference $[11, p$. 173]). The second describes the relationship between $I[u]$ and $g[u]$.

THEOREM 1. For any locally summable function $u$ in $R$ we have $g[u, R]$ $=\lim I\left[u_{h}, R_{h}\right]$.

Proof. From Lemma 1 and the definition of $g[u, R]$ follows $I\left[u_{h}, R_{h}\right]$ $\leqq \mathfrak{g}[u, R]$. Hence, once more using the definition of $\mathscr{g}[u, R]$, we have

$$
g[u, R] \leqq \lim \inf I\left[u_{h}, R_{k}\right] \leqq \lim \sup I\left[u_{h}, R_{h}\right] \leqq g[u, R],
$$

and the theorem is proved.

THEOREM 2. If $u$ is weakly differentiable, then $I[u] \leqq g[u]$, equality holding whenever $u$ is strongly differentiable.

Proof. We first require some general considerations about weakly differentiable functions $u$. Let $\alpha$ be the derivative measure of $u$. According to formulas (6), (3), and (5) in footnote 1,

$$
u_{h x}=\int K(\xi-x) d \alpha(\xi)=\int K(\xi-x) u_{x}(\xi) d \xi+\int K(\xi-x) d \theta(\xi),
$$

where $\theta$ is a singular measure. Now assume that $K$ satisfies the condition $K \leqq$ Const. $/ h^{n}$ in addition to the properties already listed (this condition is satisfied, for example, by kernels of the form $K(\xi)=K(\xi, h)=h^{-n} \tilde{K}\left(h^{-1} \xi\right)$, where $\tilde{K}$ is independent of $h$ ). Then it is easily shown( $\left.{ }^{2}\right)$ that $\lim u_{h x}=u_{x}$ al-

(2) We have $\left|u_{k z}-u_{x}\right| \leqq \int K(\xi-x)\left|u_{x}(\xi)-u_{z}(x)\right| d \xi+\int K(\xi-x) d|\theta(\xi)| \leqq\left(\right.$ Const./ $\left.h^{n}\right) \times$ $\left[\int_{X}\left|u_{x}(\xi)-u_{x}(x)\right| d \xi+|\theta|(X)\right]$, where $X$ denotes the set of points $\xi$ such that $|\xi-x| \leqq h$. The last expression tends to zero almost everywhere as $h \rightarrow 0[7$, p. 189; 11, p. 119]. 
most everywhere in $R$.

This being the case, we have by virtue of Fatou's lemma

$$
I[u, S] \leqq \lim I\left[u_{k}, S\right] \leqq \lim I\left[u_{k}, R_{k}\right],
$$

where $S$ is any compact subset of $R$. But the expression on the right equals $g[u, R]$, whatever the averaging kernel. This proves the first part of the theorem.

Next suppose that $u$ is strongly differentiable. Setting $u_{m} \equiv u$ in Lemma 1 , there results $I\left[u_{h}, R_{h}\right] \leqq I[u, R]$. Letting $h \rightarrow 0$ yields $g[u] \leqq I[u]$. In view of the earlier inequality we must therefore have $I[u]=g[u]$, and the proof is complete.

REMARK. The conclusion of Lemma 1 can now be interpreted as stating that integral averaging is a norm shrinking operation. Indeed, since $u_{k}$ is continuously differentiable, Lemma 1 and Theorem 2 imply

$$
g\left[u_{h}, R_{h}\right] \leqq g[u, R] .
$$

The same result holds for the integral $I[u]$ provided $u$ is strongly differentiable.

3. Two particular integrals. The developments of later sections will stand out in sharper relief if we first investigate the particular integrands

$$
f(p)=|p| \text { and } f(p)=\Phi(p)
$$

where $|p|$ stands for the sum of the absolute values of the components of $p$, and $\Phi(p)$ is some fixed function with the property $\Phi(p) /|p| \rightarrow \infty$ as $|p| \rightarrow \infty$. The corresponding functionals will be denoted by $D[u], D[u]$ and $E[u], \varepsilon[u]$, respectively.

TheоRем 3. $D[u]$ is finite if and only if $u$ is weakly differentiable. The set function $D[u, Q]$ is absolutely continuous if and only if $u$ is strongly differentiable.

Proof. Suppose that $u$ is weakly differentiable, and suppose also (this is tacitly assumed in the statement of the theorem) that the measure $\alpha$ associated with $u$ is finite on $R$. From the formula

$$
u_{h x}=\int K(\xi-x) d \alpha, \quad x \in R_{h},
$$

one easily infers that $D\left[u_{h}, R_{h}\right] \leqq|\alpha|(R)$. Hence by Theorem 1 ,

$$
D[u, R] \leqq|\alpha|(R)<\infty \text {. }
$$

Conversely, suppose that $D[u, R]$ is finite. We must show that $u$ is weakly differentiable, and that its measure $\alpha$ is finite on $R$. For convenience, let $C(R)$ denote the linear space whose members are real valued continuous functions with compact support in $R$. Similarly, let $C^{\prime}(R)$ denote the space of 
continuously differentiable functions with compact support in $R$. For $\omega \in C^{\prime}(R)$ let us set

$$
A(\omega)=\int_{R} u \omega_{x} d x .
$$

Evidently $A(\omega)$ is a vector-valued linear functional on $C^{\prime}(R)$. Moreover, $A$ is continuous in the uniform topology of $C(R)$. To see this, we have

$$
A(\omega)=\lim \int_{R_{h}} u_{h} \omega_{\&} d x=-\lim \int_{R_{h}} u_{h} d \omega d x,
$$

and

$$
\left|\int_{R_{\Lambda}} u_{h s \omega} d x\right| \leqq \max |\omega| \cdot \int_{R_{\Lambda}}\left|u_{h s}\right| d x .
$$

Hence by Theorem 1

$$
|A(\omega)| \leqq \max |\omega| \cdot D[u, R],
$$

proving our assertion. Since $A(\omega)$ is continuous in the topology of $C(R)$, it may evidently be extended to a continuous linear functional defined on all of $C(R)$. But then by the Riesz theorem there exists a (vector-valued) Borel measure $\alpha$ such that

$$
A(\omega)=-\int \omega d \alpha, \quad|\alpha|(R) \leqq n \Phi[u, R] .
$$

This is, however, precisely the condition that $u$ be weakly differentiable, and the first part of the theorem is proved.

The preceding work yields as a byproduct the interesting corollary

$$
D[u, R] \leqq|\alpha|(R) \leqq n D[u, R] .
$$

Hence the set function $D[u, Q]$ is absolutely continuous if and only if $u$ is weakly differentiable and its derivative measure is absolutely continuous; that is, if and only if $u$ is strongly differentiable.

THEOREM 4. If $\mathcal{E}[u]$ is finite, then $u$ is strongly differentiable.

Proof. Since $\lim E\left[u_{h}, R_{h}\right]=\varepsilon[u, R]$, the integrals $E\left[u_{h}, R_{h}\right]$ must be uniformly bounded. But then by a theorem of Nagumo, the integrals $\int\left|u_{h x}\right| d x$ are uniformly absolutely continuous. This in turn implies, by Theorem 1 , that $D[u, Q]$ is an absolutely continuous function of open sets $Q$ in $R$. According to Theorem 3 , therefore, $u$ is strongly differentiable in $R$.

Theorems 3 and $t$ throw considerable light on the type of functions which are admissible in problems of the calculus of variations. In particular, let us 
call an integrand of "area" type if it is compatible with $|p|$, and of "nonarea" type if it grows faster than any multiple of $|p|$. Then $g[u]$ will be finite in problems of area type whenever $u$ is weakly differentiable, while in problems of non-area type it is necessary that $u$ be strongly differentiable. The frequently studied quadratic variational problems, for example, are of non-area type, whence for them we should expect the admissible functions to be strongly differentiable (in this connection it is interesting to note that the usual function space approach to these problems also leads to the consideration of strongly differentiable functions as the appropriate admissibility class).

Since for non-area problems the admissible functions are necessarily strongly differentiable, there are reasonable grounds for considering either the Lebesgue integral $I[u]$ or the functional $g[u]$ as the fundamental quantity. The decision as to which one to use is, however, simplified by the fact that in most cases of practical importance both have the same value. On the other hand, it seems that there are (rather complicated) non-area integrands for which $I[u]$ and $g[u]$ differ beyond the class of Lipschitz functions. In these cases the choice of the appropriate integral must be based on aesthetic considerations. In any case, we emphasize that for area type problems the functional $\mathscr{g}[u]$ is most appropriate, since it gives a wider class of admissible functions and also because it is closer to Lebesgue's original ideas.

4. A generalization of Tonelli's theorem. In 1926 Tonelli discovered a remarkable relation between the Lebesgue area of a surface and the classical surface area integral. His work was later simplified and extended by Saks (see the discussion in Chapter $\mathrm{V}$ of reference [11]), and more recently the theory has been further refined by Cesari and Goffman. The following theorem is a generalization of all these results.

Theorem 5. Let $R$ be a bounded open set. Suppose that there exist positive constants $a$ and $A$ such that

$$
a|p| \leqq 1+f(p) \leqq A|p| \quad \text { when }|p| \geqq 1 .
$$

Then $g[u]$ is finite if and only if $u$ is weakly differentiable.

When this is the case, we have

$$
I[u] \leqq g[u]
$$

and also $g^{\prime}(x)=f\left(u_{x}\right)$ almost everywhere in $R$. In order that $I[u]=g[u]$ it is necessary and sufficient that $u$ be strongly differtntiable; and in order that this be the case it is necessary and sufficient that $\mathcal{g}[u, Q]$ be an absolutely continuous function of open sets $Q$ contained in $R$.

Proof. We may assume without loss of generality that $a \leqq 1$. Then from condition (13) it is clear that

$$
a D[u]-\text { Meas } R \leqq \mathcal{I}[u] \leqq A(D[u]+\text { Meas } R) .
$$


The first part of the theorem is therefore an immediate consequence of Theorem 3.

Now suppose $\mathscr{g}[u]$ is finite and $u$ is weakly differentiable. Inequality (14) is a consequence of Theorem 2. Moreover if $I[u]=g[u]$, then using the superadditivity of $\boldsymbol{g}$ one easily infers that $I[u, Q]=g[u, Q]$ for each open set $Q$ in $R$ whose boundary is of measure zero. Thus using (12) and (15),

$$
|\alpha|(Q)=D[u, Q] \leqq a^{-1}(I[u, Q]+\text { Meas } Q) .
$$

That is, $\alpha$ is an absolutely continuous function of open sets whose boundaries are of measure zero. Since any open set can be approximated from inside by an increasing sequence of open sets whose boundaries are of measure zero, it follows that $\alpha$ is absolutely continuous on all open sets and thence on all Borel sets. The equality $I[u]=g[u]$ thus implies that $u$ is strongly differentiable; conversely, if $u$ is strongly differentiable, then $I[u]=g[u]$ by Theorem 2.

The final statement of the theorem is obvious from what has gone before, and a detailed proof is therefore omitted.

It remains only to show that $g^{\prime}(x)=f\left(u_{x}\right)$ whenever $u$ is weakly differentiable. Let $J$ denote an arbitrary open interval in $R$. We assert that

$$
I[u, J] \leqq g[u, J] \leqq I[u, J]+\Theta(J),
$$

where $\Theta$ is a singular measure. The first half of (16) is of course obvious. To prove the second, we observe that

$$
u_{h x}=u_{x h}+\int K(\xi-x) d \theta(\xi)
$$

as in the proof of Theorem 2. Since by hypothesis the partial slopes of $f(p)$ are always less than $A$, we have

$$
f\left(u_{h x}\right) \leqq f\left(u_{x h}\right)+A\left|\int K(\xi-x) d \theta(\xi)\right| \leqq f\left(u_{x}\right)_{h}+\int K(\xi-x) d \Theta(\xi)
$$

by Jensen's inequality, where $\Theta=A|\theta|$ is a singular measure. Integrating over $J_{h}$ and applying Fubini's theorem, there results

$$
I\left[u_{h}, J_{h}\right] \leqq I[u, J]+\Theta(J) .
$$

The required inequality is now obtained by letting $h$ go to zero.

Dividing each side of (16) by $|J|$ and letting $J$ tend regularly to $x$ gives, on account of Lebesgue's differentiation theorem [11, pp. 113-119],

$$
f\left(u_{x}\right) \leqq g^{\prime}(x) \leqq f\left(u_{x}\right)
$$

almost everywhere in $E$. This completes the proof.

REMARKs. 1. When $u$ is a continuous function of two real variables $x$ and $y$ and $f=\left(1+u_{s}^{2}+u_{y}^{2}\right)^{1 / 2}$, Theorem 5 reduces to Tonelli's theorem. To see this, 
we must verify first that $g[u]$ represents the Lebesgue area of the surface $z=u(x, y)$; second, that a weakly differentiable continuous function is almost everywhere differentiable in the ordinary sense; and third, that the derivative $u_{x}=\alpha^{\prime}$ agrees almost everywhere with the ordinary derivative.

The first result is given as Theorem 2 of reference [3]; a more immediate proof can be based on Theorem 1 and the fact that for a continuous function $u$ the sequence $\left\{u_{h}\right\}$ converges uniformly on compact subsets. The second and third results are special cases of the main theorem of [14]. Indeed, in that paper it is shown that any weakly differentiable function is equivalent to a function having ordinary partial derivatives almost everywhere, and that these partial derivatives agree almost everywhere with $\alpha^{\prime}$.

2. The result of Theorem 5 implies that the set function $g[u, Q]$ can be extended to a (unique) regular Borel measure on $R\left({ }^{8}\right)$. Leaving aside for a moment the demonstration of this fact, we obtain from the Lebesgue decomposition of the resulting measure $g[u, E]$ the formula

$$
g[u, E]=\int_{E} f\left(u_{x}\right) d x+\Psi(E),
$$

where $\Psi$ is a non-negative singular measure, depending of course on the function $u$. This interesting equality may be looked upon as an alternate formulation, and in fact as a generalization, of the fundamental inequality (14).

The extension of the set function $g[u, Q]$ from open sets $Q$ to Borel sets $E$ is most easily accomplished by the general theory of generation of Borel measures ([4, pp. 231-237]; this theory is phrased so as to apply to set functions defined on compact sets, but the ideas are equally valid for functions defined on open sets). We have already observed in $\$ 1$ that $g[u, Q]$ is monotone and additive on open sets. In addition, by virtue of Theorem 1 we have easily

$$
\mathfrak{g}\left[u, Q_{1} \cup Q_{2}\right] \leqq \mathfrak{g}\left[u, Q_{1}\right]+\mathfrak{g}\left[u, Q_{2}\right],
$$

so that $g[u, Q]$ is subadditive. It only remains to show that $g[u, Q]$ is "regular," i.e. that

$$
g[u, Q]=\sup g\left[u, Q_{1}\right]
$$

where the supremum is taken over all open sets $Q_{1}$ properly contained in $Q$. But let $Q_{1}$ be an open subset of $Q$, and $C$ a compact subset of $Q_{1}$. Then

$$
\begin{aligned}
\mathfrak{g}[u, Q] & \leqq g\left[u, Q_{1}\right]+\mathfrak{g}[u, Q-C] \\
& \leqq \mathfrak{g}\left[u, Q_{1}\right]+I[u, Q-C]+\Theta(Q-C),
\end{aligned}
$$

by (16). Letting $Q_{1} \rightarrow Q$, and consequently $C \rightarrow Q$, we thus obtain

(3) The corresponding result for Lebesgue area was obtained by Verchenko, and elaborated further by Goffman (Acta Math. vol. 103 (1960)). 


$$
g[u, Q] \leqq \lim g\left[u, Q_{1}\right] .
$$

On the other hand, by the monotonicity of $\mathscr{g}[u, Q]$ we have $\mathscr{s}[u, Q]$ $\geqq \lim g\left[u, Q_{1}\right]$, and the required relation is proved.

3. When the integrand $f(p)$ depends on $p$ only through the intermediate variable $\left(p_{1}^{2}+\cdots+p_{n}^{2}\right)^{1 / 2}=\|p\|$, it is possible to give an alternate version of Theorem 5. Indeed, in this case the hypothesis (13) can be dropped in favor of the simple condition that $f$ not be identically constant. The conclusions are that if $g[u]$ is finite then $u$ is (at least) weakly differentiable, and that the second part of the theorem remains valid. To see this, we observe that if $f$ depends only on $\|p\|$, and is not identically constant, then either (13) holds, or else $f \geqq \Phi$ for some function $\Phi$ of the type considered in \$3. In either case the conclusions noted follow at once.

5. Further results. This section is devoted to certain subsidiary results and generalizations of the preceding work.

1. The basic definition of $g[u]$ can be slightly weakened by allowing sequences $\left\{u_{m}\right\}$ into competition which are only weakly convergent to $u$. That is, in place of the condition $\int_{S}\left|u_{m}-u\right| d x \rightarrow 0$ we could use simply

$$
\int\left(u_{m}-u\right) \omega d x \rightarrow 0,
$$

where $\omega$ is any continuous function with compact support in $R$. Lemma 1 remains true for this kind of convergence after only slight changes in the proof. But then Theorem 1 shows that the resulting functional is exactly the same as before.

2. The so-called inequality of Steiner for surface area has an interesting generalization to the present case. Namely, we assert that $\mathscr{g}[u]$ is a convex functional over the linear space of locally summable functions on a fixed region $R$. That is, if $u_{1}$ and $u_{2}$ are locally summable in $R$, and $u=t u_{1}+(1-t) u_{2}, 0<t<1$, then

$$
g[u] \leqq t g\left[u_{1}\right]+(1-t) g\left[u_{2}\right] .
$$

To prove this, note that since $f$ is convex in $p$,

$$
f\left(u_{h x}\right)=f\left[t u_{1 h x}+(1-t) u_{2 h x}\right] \leqq t f\left(u_{1 h x}\right)+(1-t) f\left(u_{2 h x}\right) .
$$

Integrating over $R_{h}$ and applying Theorem 1 yields (17).

It may be conjectured that if $u_{1}$ and $u_{2}$ are weakly differentiable and if $u_{1 x}=u_{2 x}$, then equality holds in (17). This conclusion is obviously legitimate if $u_{1}$ and $u_{2}$ are strongly differentiable. Moreover, a partial converse is true:

Suppose that $f$ is strictly convex and satisfies condition (13). If for some value of $t$ we have

$$
g[u]=t g\left[u_{1}\right]+(1-t) g\left[u_{2}\right],
$$


and if $\mathscr{g}[u]<\infty$, then $u_{1}$ and $u_{2}$ are weakly differentiable and their derivatives agree almost everywhere.

Proof. Since $\delta[u]<\infty$, it is evident that the functions $u, u_{1}$, and $u_{2}$ are all weakly differentiable. We now assert that the additivity relation

$$
g[u, R]=g[u, J]+g[u, \operatorname{Int}(R-J)]
$$

holds for any open interval $J$ in $R$ such that $\Theta(\partial J)=0$, irrespective of whether or not (18) holds. To see this, let $B$ denote the quantity on the right side of (19), and let $J^{*}$ denote the set of points in $R$ whose distance from $\partial J$ is less than some number $\epsilon>0$. Then clearly

$$
\leftrightarrow \leqq \mathscr{g}[u, R] \leqq \leftrightarrow+\mathscr{g}\left[u, J^{*}\right] .
$$

But by the argument proving (16) we have

$$
g\left[u, J^{*}\right] \leqq I\left[u, J^{*}\right]+\Theta\left(J^{*}\right),
$$

whence $\lim _{\epsilon \rightarrow 0} g\left[u, J^{*}\right]=\Theta(\partial J)=0$. This proves our assertion.

It follows from (17), (18), (19), and the superadditivity of $g$ that

$$
g[u, J]=t g\left[u_{1}, J\right]+(1-t) g\left[u_{2}, J\right] \quad(\Theta(\partial J)=0) .
$$

Also, the set of intervals $J$ such that $\Theta(\partial J)=0$ is certainly dense in the set of all intervals. We may therefore form the set-theoretic derivative of (20), whence by Theorem 5

$$
f\left(u_{x}\right)=t f\left(u_{1 x}\right)+(1-t) f\left(u_{2 x}\right)
$$

almost everywhere in $R$. Since $u_{x}=t u_{1 x}+(1-t) u_{2 x}$ almost everywhere, the strict convexity of $f$ implies $u_{1 x}=u_{2 x}=u_{x}$, as required.

The preceding discussion is based upon, and generalizes, a corresponding result of McShane for the surface area functional.

\section{PART II}

6. Integrands of type I and II. We now turn to a more general class of integrands for which the results of the preceding sections remain valid. An integrand $f(x, u, p)$ will be called of type $\mathrm{I}$ if for any two points $x$ and $y$ in $R_{0}$, and for all values of $u, v$, and $p$, we have

$$
|f(x, u, p)-f(y, v, p)| \leqq \lambda(|x-y|)[1+f(x, u, p)]+\mu(|u-v|) .
$$

Here $\lambda(\sigma)$ and $\mu(\sigma)$ are moduli of continuity, with $\mu$ satisfying $\mu(\sigma) \leqq$ Const. $\sigma$ for large values of $\sigma$. An integrand will be called of type II if, similarly,

$$
|f(x, u, p)-f(y, v, p)| \leqq \lambda(|x-y|+|u-v|)[1+f(x, u, p)] .
$$

Important examples of integrands of type I and type II are

$$
f=f(x, p)=A(x) F(p), \quad f=f(x, u, p)=A(x, u) F(p),
$$


where $A(x)$ and $A(x, u)$ are continuous functions with some positive lower bound. Finally, an integrand will be called of type $\mathrm{II}_{B}$ if the relevant inequality holds for all $u, v$ bounded in absolute value by $B$.

The following two lemmas play the same role for our present class of integrands as Lemma 1 did for the integrand $f(p)$. In these results, and throughout the rest of the paper, it will be tacitly assumed that the regions $R$ under consideration are subsets of the basic domain of continuity $R_{\mathbf{0}}$. We recall that $R_{m}$ denotes the domain of definition of a function $u_{m}$.

LEMMA 2. Suppose $f$ is of type I, and let $u$ and $\left\{u_{m}\right\}$ satisfy the hypotheses of Lemma 1. Then for every compact subset $S$ of $R$ we have

$$
\lim \sup I\left[u_{h}, S\right] \leqq \lim \inf I\left[u_{m}, R_{m}\right] .
$$

Proof. Let $S_{0}$ be some fixed compact subset of $R$, containing $S$ in its interior. We suppose that $S_{0} \subset R_{m}$ for all $m \geqq m_{0}$, and also that $S \subset S_{0 \mathrm{ph}}$ for all $h \leqq h_{0}$. Then, restricting consideration to such values of $m$ and $h$, we find as in Lemma 1 that for all $x \in S$,

$$
\begin{aligned}
f\left(x, u_{k}, u_{k x}\right) & \leqq f\left(x, u_{k}, u_{m x k}\right)+\epsilon \\
& \leqq \int K(\xi-x) f\left[x, u_{h}(x), u_{m x}(\xi)\right] d \xi+\epsilon \\
& =\int K(\xi-x) f\left[\xi, u_{m}(\xi), u_{m x}(\xi)\right] d \xi+J+\epsilon,
\end{aligned}
$$

where $J$ denotes the difference of the integrals on the preceding two lines, and $\epsilon=\epsilon(m, h)$ tends to zero as $m \rightarrow \infty$. Integrating over $S$ and applying Fubini's theorem to the first term on the right easily yields

$$
I\left[u_{h}, S\right] \leqq I\left[u_{m}, R_{m}\right]+\int_{S} J d x+\epsilon \text { Meas } S .
$$

We must now estimate the integral of $J$. Since $f$ is of type $\mathrm{I}$, one sees that

$$
\begin{aligned}
|J| & \leqq \int K(\xi-x)\left[\lambda(|\xi-x|)\left\{1+f\left[\xi, u_{m}(\xi), u_{m x}(\xi)\right]\right\}+\mu\left(\left|u_{m}-u_{h}\right|\right)\right] d \xi \\
& \leqq \lambda(h)\left\{1+f\left(x, u_{m}, u_{m x}\right)_{h}\right\}+\int K(\xi-x) \mu\left(\left|u_{m}-u_{h}\right|\right) d \xi .
\end{aligned}
$$

In virtue of the hypothesis $\mu \leqq$ Const. $\sigma$ for large $\sigma$, there is no loss of generality in assuming that $\mu$ is concave (convex downwards). Thus again using Fubini's theorem and Jensen's inequality, we have

$$
\left|\int_{S} J d x\right| \leqq \lambda(h)\left\{\text { Meas } S+I\left[u_{m}, R_{m}\right]\right\}+\mu(P / \text { Meas } S) \cdot \text { Meas } S,
$$

where 


$$
\begin{aligned}
P & =\int_{s} \int K(\xi-x)\left|u_{m}(\xi)-u_{h}(x)\right| d \xi d x \\
& \leqq \int_{S_{0}}\left|u_{m}-u\right| d \xi+\int_{S} \int K(\xi-x)|u(\xi)-u(x)| d \xi d x+\int_{S}\left|u-u_{h}\right| d x .
\end{aligned}
$$

The middle integral can be written in the form $\int K(\xi) T(\xi) d \xi$, where

$$
T(\xi)=\int_{S}|u(x+\xi)-u(x)| d x .
$$

It is well known that $T(\xi)$ tends to zero as $\xi \rightarrow 0$. Therefore $P \leqq \epsilon_{1}+\epsilon_{2}$, where $\epsilon_{1}=\epsilon_{1}(m)$ and $\epsilon_{2}=\epsilon_{2}(h)$ tend to zero as $m$ and $h$ tend respectively to infinity and zero.

Inserting the estimates of the previous paragraph into inequality (21), there results

$$
I\left[u_{h}, S\right] \leqq\{1+\lambda(h)\} I\left[u_{m}, R_{m}\right]+\left\{\epsilon+\lambda(h)+\mu\left(\frac{\epsilon_{1}+\epsilon_{2}}{\text { Meas } S}\right)\right\} \text { Meas } S .
$$

Letting $m \rightarrow \infty$ through an appropriate subsequence now yields (22) $I\left[u_{h}, S\right] \leqq\{1+\lambda(h)\} \lim \inf I\left[u_{m}, R_{m}\right]+\left\{\lambda(h)+\mu\left(\epsilon_{2} /\right.\right.$ Meas $\left.\left.S\right)\right\}$ Meas $S$.

To complete the proof of the lemma, it is only necessary to let $h$ tend to zero through an appropriate subsequence.

Lemma 3. Let $f$ be of type II. Suppose that $u$ is continuous, but otherwise let $u$ and $\left\{u_{m}\right\}$ satisfy the conditions of Lemma 1. Then for every compact subset $S$ of $R$ we have

$$
\lim \sup I\left[u_{h}, S\right] \leqq \lim \inf I\left[u_{m}, R_{m}\right]
$$

Proof. We may assume without loss of generality that the sequence $\left\{u_{m}\right\}$ converges uniformly to $u$ in any compact subset $S$ of $R$. Indeed, if $\left\{u_{m}\right\}$ did not converge uniformly, we could construct for any $\epsilon>0$ another sequence $\left\{v_{m}\right\}$ which converges uniformly, and such that

$$
\lim I\left[v_{m}, R_{m}\right] \leqq \lim \inf I\left[u_{m}, R_{m}\right]+\epsilon .
$$

The construction of the alternate sequence $\left\{v_{m}\right\}$ can be carried out as in $[13$, pp. 30-31], the only difference being that the functions $v_{m}$ which arise are now strongly differentiable rather than piecewise continuously differentiable.

This much being shown, the rest of the proof is very nearly the same as the proof of Lemma 2, except in some respects simpler. We shall omit the details. The next two results are parallel to Theorems 1 and 2.

Theorem 6. Suppose that $R$ is bounded. If $f$ is of type I and $u$ is summable over $R$, then $g[u, R]=\lim I\left[u_{h}, R_{h}\right]$. If $f$ is of type II and $u$ is uniformly con- 
tinuous over $R$, then again $g[u, R]=\lim I\left[u_{h}, R_{h}\right]$.

Proof. In the first case, by letting $S \rightarrow R_{h}$ in (22) we obtain

$I\left[u_{h}, R_{h}\right] \leqq\{1+\lambda(h)\} \lim \inf I\left[u_{m}, R_{m}\right]+\left\{\lambda(h)+\mu\left(\epsilon_{2}^{\prime} /\right.\right.$ Meas $\left.\left.S\right)\right\}$ Meas $R$, where

$$
\epsilon_{2}^{\prime}=\int_{R_{h}}\left|u-u_{h}\right| d x+\operatorname{Max}_{|\xi| \leqq h} \int_{R_{h}}|u(x+\xi)-u(x)| d x .
$$

Since $u$ is summable over $R$, the right hand side of (24) tends to zero as $h \rightarrow 0$. Therefore by (23)

$$
\lim \sup I\left[u_{h}, R_{h}\right] \leqq \lim \inf I\left[u_{m}, R_{m}\right] .
$$

The formula $g[u, R]=\lim I\left[u_{h}, R_{h}\right]$ follows at once from this inequality, exactly as in the proof of Theorem 1 . The second part of Theorem 6 is treated in much the same way, except that the details are simpler.

THEOREM 7. Let $f$ be of type I. Then for any weakly differentiable function $u$ we have $I[u] \leqq g[u]$, with equality holding whenever $u$ is strongly differentiable. If $f$ is of type II, the same result holds provided $u$ is also continuous.

Proof. By Fatou's lemma (see the proof of Theorem 2), and by either Lemma 2 or Lemma 3, we have

$$
I[u, S] \leqq \lim I\left[u_{h}, S\right] \leqq \lim \inf I\left[u_{m}, R_{m}\right] .
$$

The inequality $I[u] \leqq \mathscr{g}[u]$ is an immediate consequence of (25).

Now suppose that $u$ is strongly differentiable. If we set $u_{m} \equiv u$ in Lemma 2 or Lemma 3, there results

$$
\lim \sup I\left[u_{h}, S\right] \leqq I[u, R] .
$$

Let $\left\{R_{\nu}\right\}$ be a sequence of open sets with compact closure in $R$, such that $R_{\nu} \rightarrow R$ as $\nu \rightarrow \infty$. In virtue of (26), there exists for each integer $\nu$ a corresponding number $h=h(\nu) \leqq 1 / \nu$ such that

$$
I\left[u_{h(v)}, R_{r}\right] \leqq I[u, R]+1 / \nu .
$$

Letting $\nu \rightarrow \infty$ and using the definition of $g$ yields $g[u] \leqq I[u]$, and the proof is complete.

REMARK. The result of Lemma 3, and the parts of Theorems 6 and 7 which refer to integrands of type II, can be extended to apply to integrands of type $\mathrm{II}_{B}$. In particular, if $|u|<B$ in $R$ then it is easily seen that all the proofs remain essentially unchanged. We shall make use of this fact in the proof of Theorem 9.

7. Three lemmas. The deeper considerations of the following sections depend on several technical approximation lemmas which it is convenient to present at this time. 
Let $f(t, p)$ be a continuous function on the product space $\Omega \times E^{n}$, where $\Omega$ is an open set in the $N$ dimensional space $E^{N}$. Suppose also that

$$
f \geqq 0, \quad f \text { convex in } p .
$$

If $f$ has continuous partial derivatives $f_{\boldsymbol{p}}$, we define

$$
F(t, p, q)=f(t, q)+(p-q) \cdot f_{p}(t, q)
$$

and, for fixed $L>0$,

$$
f_{L}(t, p)=\underset{|q| \leqq L}{\text { Supremum }}\{0, F(t, p, q)\} .
$$

These functions have a simple geometric interpretation when $t$ is held fixed: In the $(n+1)$-dimensional $(p, z)$ space, the equation $z=F(t, p, q)$ represents the tangent plane to the figurative surface $z=f(t, p)$ at the point $(q, f(t, q))$. Similarly, the equation $z=f_{L}(t, p)$ represents essentially the envelope of a set of tangent planes of the figurative surface. The following lemma describes certain useful properties of $f_{L}$.

Lemma 4. The function $f_{L}(t, p)$ defined above has the following properties:

(i) $f_{L}$ is continuous in $\Omega \times E^{n}$, convex in $p$, and $f_{L} \geqq 0$.

(ii) $f_{L} \leqq f$, equality holding for all $|p| \leqq L$.

(iii) For every compact subset $\Sigma$ of $\Omega$ there exists a constant $A$ and a modulus of continuity $\lambda(\sigma)$ such that

$$
\begin{aligned}
f_{L}(t, p) & \leqq A(1+|p|), \\
\left|f_{L}(s, p)-f_{L}(t, p)\right| & \leqq \lambda(|s-t|) \cdot(1+|p|) .
\end{aligned}
$$

Proof. Obviously $f_{L} \geqq 0$. The convexity of $f_{L}$ is likewise clear, since $f_{L}$ is the supremum of a family of linear functions of $p$. Also, $f_{L}$ is continuous, since $F(t, p, q)$ is uniformly continuous over any compact subset of $\Omega \times E^{n} \times E^{n}$. This proves property (i). Condition (ii) is evident from the geometrical nature of the construction, or else follows analytically from the inequality $F(t, p, q)$ $\leqq f(t, p)$. Finally, since uniform inequalities of the form (iii) hold for each function $F(t, p, q)$ when $|q| \leqq L$ and $s$ and $t$ are in $\Sigma$, the same inequalities must hold for the supremum of these functions.

Lemma 5. Let $f(t, p)$ be continuous on $\Omega \times E^{n}$, and satisfy (27). Then for every pair of positive numbers $L$ and $\epsilon$ and every compact subset $\Sigma$ of $\Omega$, there exists a function $g(t, p)$ with compact support in $\Omega$, satisfying the same hypotheses as $f$, and such that

(i) $g \leqq f+\epsilon(1+|p|)$.

(ii) $|g-f| \leqq \epsilon$ for $t \in \Sigma$ and $|p| \leqq L$.

(iii) There exist constants $A$ and $C$ such that

$$
\begin{aligned}
g(t, p) & \leqq A(1+|p|), \\
|g(s, p)-g(t, p)| & \leqq C|s-t|(1+|p|) .
\end{aligned}
$$


Proof. In order to construct the function $g$ we shall first need to introduce a certain integral average of the function $f$. For any real number $h>0$ let us define

$$
f_{(h)}=f_{(k)}(t, p)=\int K(\tau-t, \xi-p) f(\tau, \xi) d \tau d \xi,
$$

where $K(\tau, \xi)$ is an averaging kernel of the type described in $\$ 1$. We suppose that $h$ is so small that

$$
\left|f_{(h)}-f\right| \leqq \epsilon \quad \text { for } t \in \Sigma_{0} \text { and }|p| \leqq L+1,
$$

where $\Sigma_{0}$ is some fixed compact subset of $\Omega$ which contains $\Sigma$ in its interior. Let $\alpha(t)$ be a continuously differentiable function with support in $\Sigma_{0}$, such that $0 \leqq \alpha \leqq 1$ everywhere and $\alpha \equiv 1$ in $\Sigma$. We then set

$$
g(t, p) \equiv \alpha(t) f_{(h) L}(t, p) .
$$

By Lemma 4, $g$ satisfies the same hypothesis as $f$. Moreover, for $t \in \Sigma$ and $|p| \leqq L$ we have $g=f_{(h) L}=f_{(h)}$, so that condition (ii) holds in virtue of (28). Also by Lemma $4, f_{(k) L}$ satisfies (iii) when $t \in \Sigma_{0}$ (recall that $f_{(k)}$ is differentiable with respect to $t$, so that the modulus $\lambda$ can be assumed Lipschitzian). Multiplication by $\alpha(t)$ does not affect the validity of (iii) beyond possibly changing the constants. It remains only to verify condition (i). We have

$$
\begin{array}{lr}
f_{(h) L} \leqq f_{(h)} \leqq f+\epsilon & \text { for } t \in \Sigma_{0},|p| \leqq L+1, \\
f_{(h) L}=f_{(h)} \geqq f-\epsilon & \text { for } t \in \Sigma_{0},|p| \leqq L .
\end{array}
$$

The first inequality implies (i) in the range $|p| \leqq L+1$. On the other hand, for $|p| \geqq L+1$ we have

$$
\begin{array}{ll}
g \leqq f_{(h) L} \leqq f+2 \epsilon|p|, & t \in \Sigma_{0}, \\
g=0, & t \in \Sigma_{0} .
\end{array}
$$

(The first inequality of (29) may be obtained most simply by considering the values of the functions $f_{(h) L}$ and $f$ along a "generator" of $f_{(h) L}$ passing through the point $p$ : the values of $f_{(h) L}$ are linear and those of $f$ convex.) Making the inessential replacement of $\epsilon$ by $\epsilon / 2$ completes the proof.

REMARKs. For later use we shall need two slightly different versions of Lemma 5.

1. Suppose that the function $f$ of Lemma 5 has the additional property that for every compact subset of $\Omega$ there exists a constant $a$ such that $1+f$ $\geqq a|p|$. Then we can replace condition (i) in Lemma 5 by

$$
g \leqq f+\epsilon(1+f),
$$

this being evident for $t \in \Sigma_{0}$, and obviously true elsewhere since $g=0$ outside $\Sigma_{0}$. Similarly, the second part of (iii) implies the further result

$$
|g(s, p)-g(t, p)| \leqq D|s-t|\{1+g(t, p)\}
$$


for all $s$ and $t$ in $\Sigma$, and for $L$ sufficiently large.

2. If $f$ is strictly convex in $p$, then we can derive in place of (29) the stronger conclusion

$$
f_{(h) L} \leqq \operatorname{Max}[0, f-k(|p|-L-1)+2 \epsilon|p|], \quad t \in \Sigma_{0},|p| \geqq L+1,
$$

where $\alpha$ is some positive constant. Thus by suitably adjusting the number $\epsilon$, $\epsilon<\alpha / 2$, it is possible to replace condition (i) by $g \leqq f+\epsilon$, and a fortiori by (i)'.

The remainder of the section concerns the approximation of $I[u]$ by integrals of continuously differentiable functions. Such an approximation can by no means be taken for granted (cf. $[16$, p. 212]).

LEMMA 6. Suppose that there exists a constant $A$ and a function $\gamma(p) \geqq 0$ such that

$$
\gamma(p) \leqq f(x, u, p) \leqq A(1+|p|+\gamma(p)) .
$$

Then for every weakly differentiable function $u$ in $R$, and for every compact subset $S$ of $R$, we have $\lim \int_{S} f\left(x, u_{h}, u_{x h}\right) d x=I[u, S]$.

Proof. If $I[u, S]=\infty$ the conclusion is a consequence of Fatou's lemma. Hence it can be assumed that $I[u, S]<\infty$. By application of Jensen's inequality and the hypothesis (30), one has for $x \in S$,

$$
\begin{aligned}
f\left(x, u_{h}, u_{x h}\right) & \leqq \int K(\xi-x) f\left[x, u_{h}(x), u_{x}(\xi)\right] d \xi \\
& \leqq A\left[1+\left|u_{x}\right|_{h}+f\left(x, u, u_{x}\right)_{h}\right] .
\end{aligned}
$$

Since $\left|u_{x}\right|_{h} \rightarrow\left|u_{x}\right|$ and $f_{h} \rightarrow f$, the integrals $\int\left|u_{x}\right|_{h} d x, \int f_{h} d x$, are uniformly absolutely continuous $\left[4\right.$, p. 100]. It follows that the integral $\int f\left(x, u_{h}, u_{x h}\right) d x$ is also uniformly absolutely continuous.

We next observe that the functions $u_{h}$ and $u_{x h}$ converge locally to $u$ and $u_{x}$, respectively. Consequently, $f\left(x, u_{h}, u_{x h}\right)$ converges in measure to $f\left(x, u, u_{x}\right)$. But this together with the result of the preceding paragraph implies

$$
\lim \int_{S} f\left(x, u_{h}, u_{x h}\right) d x=\int_{S} f\left(x, u, u_{x}\right) d x,
$$

(cf. $[4$, p. 108]). This completes the proof. As a corollary of Lemma 6, we have the following important result.

THEOREM 8. If $u$ is Lipschitzian, or if $u$ is strongly differentiable and $f$ satisfies the hypothesis of Lemma 6 , then $\Im[u] \leqq I[u]$.

Proof. Consider first the case when $u$ is strongly differentiable. Since $u_{h x}=u_{x h}$, Lemma 6 implies

$$
\lim I\left[u_{h}, S\right]=I[u, S] \leqq I[u, R] .
$$


The conclusion $g[u, R] \leqq I[u, R]$ follows easily. Next, if $u$ is Lipschitz continuous, it is surely strongly differentiable with bounded derivatives in any compact $S$. The conclusion of Lemma 6 can therefore be obtained without auxiliary conditions restricting the behavior of $f$ for large $p$. The remainder of the proof is the same as before.

8. Normal integrands. In this section we shall consider the important class of integrands which have the property that for each fixed $(x, u)$

$$
f(x, u, p) \rightarrow \infty \quad \text { as }|p| \rightarrow \infty .
$$

Such integrands will here be called normal. Since by assumption $f$ is convex in $p$ and continuous, a necessary and sufficient condition that $f$ be normal is that for every compact set of values of $x$ and $u$, there exists a corresponding positive constant $a$ such that $a|p| \leqq 1+f$.

THEOREM 9. Let $f$ be normal. Then for every continuous weakly differentiable function $u$ we have $I[u] \leqq g[u]$, equality holding whenever $u$ is Lipschitzian. If $f$ is independent of $u$, we can drop the hypothesis that $u$ be continuous.

Though we shall make no use of this fact, it may be remarked here that a function is continuous and weakly differentiable if and only if it is of bounded variation in the sense of Tonelli; cf. $[5 ; 14]$.

Proof of Theorem 9. Let $Q$ be a fixed open set with compact closure in $R$. Let $B$ be a bound on the function $u$ in $Q$, and let $\epsilon$ be a small positive number. By Lemma 5 and by the remark following the proof of that lemma, there exists an integrand $g(x, u, p)$ of type $\mathrm{II}_{B}$ in $Q$ such that

$$
\begin{aligned}
g & \leqq f+\epsilon(1+f) \\
|g-f| & \leqq \epsilon
\end{aligned}
$$$$
\text { for }|u| \leqq B, \text { and }|p| \leqq 1 / \epsilon .
$$

Applying Theorem 7 to the integrand $g$, one finds

$$
I_{o}[u, Q] \leqq g_{o}[u, Q] \leqq(1+\epsilon) \mathscr{g}[u, R]+\epsilon \text { Meas } Q .
$$

By property (ii), $\lim _{\epsilon \rightarrow 0} g\left(x, u, u_{x}\right)=f\left(x, u, u_{x}\right)$ almost everywhere in $Q$. Therefore by Fatou's lemma $I[u, Q] \leqq g[u, R]$. Letting $Q \rightarrow R$ gives the inequality of Theorem 9. Moreover, in consequence of Theorem $8, I[u]=g[u]$ whenever $u$ is Lipschitzian.

When $f=f(x, p)$ the proof is very similar, except that the function $g(x, p)$ is now of type I. The following result represents our ultimate generalization of Tonelli's theorem.

TheOREM 10. Let $R$ be a bounded open region. Suppose that there exist constants $a$ and $A$ such that

$$
a|p| \leqq 1+f(x, u, p) \leqq A|p| \quad \text { when }|p| \geqq 1 .
$$

Then $g[u]$ is finite if and only if $u$ is weakly differentiable. 
When this is the case, and when, further, $u$ is continuous if $f$ depends on $u$, we have

$$
\mathscr{g}[u] \geqq I[u]
$$

and $\mathfrak{g}^{\prime}(x)=f\left(x, u, u_{x}\right)$ almost everywhere in $R$. In order that $g[u]=I[u]$ it is necessary and sufficient that $u$ be strongly differentiable; and in order that this be the case it is necessary and sufficient that $\Phi[u, Q]$ be an absolutely continuous function of open sets $Q$ in $R$.

Proof. Except for the formula $g^{\prime}=f$, everything is just as in the proof of Theorem 5, with the basic inequality (31) now following from Theorems 8 and 9 rather than from Theorem 2.

To prove that $\mathfrak{g}^{\prime}=f$ it is enough (as in the proof of Theorem 5) to show that for every open interval $J$ in $R$

$$
g[u, J] \leqq I[u, J]+\Theta(J) .
$$

Clearly

$$
f\left(x, u_{h}, u_{h x}\right) \leqq f\left(x, u_{h}, u_{x h}\right)+A\left|\int K(\xi-x) d \theta(\xi)\right| .
$$

Integrating over $J_{h}$ easily yields

$$
I\left[u_{h}, J_{h}\right] \leqq \int_{J} f\left(x, u_{h}, u_{x h}\right) d x+\Theta(J),
$$

where $\Theta$ is the singular measure $A|\theta|$. Now let $h \rightarrow 0$. The left side of (33) certainly tends to a number greater than or equal to $g[u, J]$. The first term on the right tends, by Lemma 6 , to $I[u, J]$. This proves (32), and thus also Theorem 10.

\section{PART III}

9. General considerations. Lower semicontinuity. There are many integrands which do not satisfy the conditions of the preceding sections, but which nevertheless are amenable to an alternate approach. It is the purpose of the present part to investigate the functions $I[u]$ and $g[u]$ corresponding to these more difficult integrands. The major interest will be in determining conditions guaranteeing that $\mathscr{g}[u]=I[u]$ for strongly differentiable functions.

As we have seen, this involves two essentially different considerations. Namely, one must show on the one hand that $g[u] \leqq I[u]$ and on the other that $I[u] \leqq g[u]$. A moment's reflection will show that the former inequality is connected with the problem of approximating $I[u]$ by integrals $I\left[u_{m}\right]$ of continuously differentiable functions. The best result available in this respect is Lemma 6, with its attendant corollary, Theorem 8 . This being the case, we shall turn our full attention to the latter inequality $I[u] \leqq g[u]$. The 
reader has no doubt observed the close relation between this inequality and the traditional lower semicontinuity properties of variational integrals. Indeed, the inequality $I[u] \leqq g[u]$ is itself a type of lower semicontinuity theorem, while, conversely, certain lower semicontinuity theorems imply the inequality. It thus becomes evident that our goal must be to obtain as strong lower semicontinuity theorems as possible: These results, in conjunction with Theorem 8, will provide us with the required relations between $I[u]$ and $\delta[u]$. In particular, since $g[u] \leqq I[u]$ for any continuously differentiable function, a lower semicontinuity theorem immediately implies the consistency of $\&[u]$ and $I[u]$ for the corresponding integrand.

Before stating our main results, we shall for completeness first set down the lower semicontinuity theorems implied by the preceding work.

THEOREM 11. Suppose that $f$ is of type I. Let $u$ be weakly differentiable in $R$, and let $\left\{u_{m}\right\}$ be a seguence of strongly differentiable functions which are locally convergent to $u$ in $R$. Then

$$
I[u, R] \leqq \lim \inf I\left[u_{m}, R_{m}\right] .
$$

If $f$ is independent of $u$, we may replace local convergence by weak convergence in the sense of $\S 5$.

Suppose that $f$ is of type II. Let $u$ be a continuous weakly differentiable function in $R$, and let $\left\{u_{m}\right\}$ be a sequence of strongly differentiable functions which are convergent in measure to $u$ in $R$. Then

$$
I[u, R] \leqq \lim \inf I\left[u_{m}, R_{m}\right] .
$$

Proof. For local convergence, these results follow at once from Lemma 2 and Lemma 3, respectively, and Fatou's lemma. The more general convergence allowed in the statement of the theorem is due to the fact that Lemmas 2 and 3 actually hold for the type of convergence noted here. The main results of Part III may now be stated:

THEOREM 12. Suppose that $f=f(x, u, p)$ satisfies any one of the following conditions:
A. $f$ is normal.
B. $f$ is strictly convex.
C. The derivatives $f_{x}, f_{p}$, and $f_{p x}$ exist and are continuous.

Then on the class of strongly differentiable functions the integral $I[u]$ is lower semicontinuous with respect to local convergence in $L_{1}$.

THEOREM 13. $I[u]$ is lower semicontinuous with respect to local convergence in $L_{1}$ on any class of strongly differentiable functions uniformly satisfying the inequality $D[u] \leqq H<\infty$.

The following three sections of the paper are devoted to the proof of Theorems 12 and 13.

REMARKs. As we have already noted in the introduction, Theorems 11 through 13 are generalizations of well-known lower semicontinuity theorems 
of Tonelli and Morrey. Other related results are due to $\mathrm{S}$. Cinquini and $\mathrm{L}$. Giuliano.

In consequence of Theorem 12 it is apparent that $I[u] \leqq \mathscr{g}[u]$ for any integrand of type $\mathrm{A}, \mathrm{B}$, or $\mathrm{C}$, and any strongly differentiable function $u$. Furthermore, if $u$ is Lipschitzian then $I[u]=\Phi[u]$.

Finally, because the hypotheses on $f$ in Theorem 12 are very weak, one might suspect that the conclusion holds with no restrictions on $f$ beyond those formulated at the outset of the paper. This is, however, not the case. There do in fact exist non-negative convex integrands for which semicontinuity fails for local convergence. Such an example (necessarily fairly complicated) was constructed by Aronszajn and appears in reference [10]. On the other hand, Theorem 13 shows that if certain restrictions are placed on the functions $u$, then lower semicontinuity does indeed hold for general integrands.

10. Preliminary lemmas. It is convenient to begin with some simple lemmas, the first of which generalizes a well-known result of Tonelli.

LEMMA 7. Let $S$ be a compact set in $E^{n}$, and define

$$
\mathcal{K}[u]=\int_{\delta} Q(x, u) \cdot u_{x} d x,
$$

where $Q$ is a continuous vector-valued function with support in $S$. Suppose there exist constants $M$ and $M^{\prime}$ such that

$$
|Q| \leqq M, \quad|Q(x, u)-Q(y, u)| \leqq M^{\prime}|x-y| .
$$

Then for every pair of strongly differentiable functions $u$ and $v$ which are defined on some open set containing $S$, we have

$$
|\mathfrak{K}[u]-\mathscr{K}[v]| \leqq n M^{\prime} \int_{S}|u-v| d x .
$$

Proof. Suppose first that $Q^{\circ}$ is differentiable with respect to $x$, and that $\left|Q_{x}\right| \leqq M^{\prime}$. Setting

$$
P(x) \equiv \int_{u_{h}}^{v_{h}} Q(x, \sigma) d \sigma, \quad x \in S,
$$

we find by the divergence theorem

Thus

$$
0=\int_{S} \nabla \cdot P d x=\varkappa\left[v_{h}\right]-\varkappa\left[u_{h}\right]+\int_{S} \int_{u_{k}}^{v_{h}} \nabla_{z} \cdot Q d x .
$$

$$
\left|\varkappa\left[u_{h}\right]-\varkappa\left[v_{h}\right]\right| \leqq n M^{\prime} \int_{S}\left|u_{h}-v_{h}\right| d x .
$$

Letting $h \rightarrow 0$ and applying Lemma 6 yields (34). The result for nondifferentiable $Q$ is easily reduced to this one by smoothing $Q$ with respect to the variable $x$. 
Lemma 8. Let $f$ satisfy any one of the hypotheses A, B, or C of Theorem 12. Then for every $\epsilon>0$ and every compact subset $S$ of $R$ there exists a function $g=g(x, u, p)$ with the properties

(i) $g \leqq f+\epsilon(1+f)$,

(ii) $|g-f| \leqq \epsilon$ for $x \in S,|u|+|p| \leqq 1 / \epsilon$.

(iii) $g$ is continuous, convex in $p$, and $g \geqq 0$. Furthermore, $g$ has compact support in $x$ and $u$.

(iv) $g$ has continuous partial derivatives $g_{p}$ which are Lipschitz continuous in $x$ and $p$ and satisfy $\left|g_{p}\right| \leqq M$ for some constant $M$.

Proof. Consider first the case when $f$ satisfies hypothesis A or B. By Lemma 5 and the remarks following this lemma, there exists a function $\boldsymbol{g}^{\prime}$ satisfying conditions (i)-(iii), Lipschitz continuous in $x$, and such that $g^{\prime} \leqq A(1+|p|)$. Averaging this function with respect to $p$ does not alter the validity of (i)-(iii), while at the same time (iv) becomes true with $M=n A$.

In case $C$, let $\alpha=\alpha(x, u)$ be a function with compact support in $R \times E^{\mathbf{1}}$, satisfying $0 \leqq \alpha \leqq 1$, and such that $\alpha \equiv 1$ when $x \in S$ and $|u| \leqq 1 / \epsilon$. We set

$$
g^{\prime}(x, u, p)=\alpha(x, u) f_{L}(x, u, p), \quad L=1 / \epsilon .
$$

By Lemma 4 , conditions (i)-(iii) are satisfied by $g^{\prime}$. Furthermore, $g^{\prime} \leqq A(1+|p|)$ and $g^{\prime}$ is Lipschitz continuous in $x$. Smoothing $g^{\prime}$ with respect to $p$ completes the proof.

11. Proof of Theorem 12. Suppose to begin with that lower semicontinuity has been established for integrands $g$ which satisfy conditions (iii) and (iv) of Lemma 8. We shall show that this implies the truth of Theorem 12.

For let $f$ be an integrand satisfying the hypotheses of Theorem 12, let $S$ be an arbitrary compact subset of $R$, and let $\epsilon>0$ be a small constant. Let $g$ denote the integrand associated to $f, S$, and $\epsilon$ by Lemma 8 ; in view of condition (iii) we may suppose that $g$ vanishes outside some compact subset $S_{0}$ of $R$. Then by our initial assumption and by property (i) of Lemma 8 , if $u_{m} \rightarrow u$,

$$
I_{o}[u, S] \leqq \lim \inf I_{\theta}\left[u_{m}, R_{m}\right] \leqq(1+\epsilon) \lim \inf I\left[u_{m}, R_{m}\right]+\epsilon \operatorname{Meas} S_{0} .
$$

Now let $\epsilon \rightarrow 0$. By virtue of (ii) and Fatou's lemma $I[u, S] \leqq \lim I_{0}[u, S]$, whence we obtain from (35),

$$
I[u, S] \leqq \lim \inf I\left[u_{m}, R_{m}\right] .
$$

Letting $S \rightarrow R$ completes the proof of Theorem 12, subject of course to the initial assumption concerning $g$.

It remains, then, to show that $I_{\theta}[u]$ is lower semicontinuous. Let $S_{0}$ denote the support of $g$ in $R$, and let $\epsilon$ be a given small number. Let us fix $h$ so small that $u_{h}$ is defined in $S_{0}$ and 


$$
\int_{s_{0}}\left|u_{x}-u_{x h}\right| d x \leqq \epsilon .
$$

Now the Weierstrass $E$-function associated with the integrand $g$ is defined by

$$
E(x, u, p, q)=g(x, u, q)-g(x, u, p)-(q-p) \cdot g_{p}(x, u, p) .
$$

Since $g$ is convex and $\left|g_{p}\right| \leqq M$, it is apparent that $0 \leqq E(x, u, p, q) \leqq 2 M|p-q|$. Therefore, if we make the abbreviations $u_{x}=p, u_{m x}=p_{m}$, we have for all $x \in S_{0}$ and all $m$ suitably large

$$
\begin{aligned}
g\left(x, u_{m}, p_{m}\right)- & g(x, u, p) \\
\geqq & g\left(x, u_{m}, p_{h}\right)+\left(p_{m}-p_{h}\right) \cdot g_{p}\left(x, u_{m}, p_{h}\right) \\
& -g\left(x, u, p_{h}\right)-\left(p-p_{h}\right) \cdot g_{p}\left(x, u, p_{h}\right)-2 M\left|p-p_{h}\right| .
\end{aligned}
$$

Consider the function $Q(x, u) \equiv g_{p}\left[x, u, p_{h}(x)\right]$. By hypothesis $g_{p}(x, u, p)$ is Lipschitz continuous in $x$ and $p$. Moreover, since $g$ has compact support in $x$ and $u$ the Lipschitz coefficient is uniformly bounded over any set of points $(x, u, p)$ for which $|p|$ is uniformly bounded. It follows that there exists a constant $M^{\prime}$, depending at most on the previously fixed number $h$, such that

$$
|Q(x, u)-Q(y, u)| \leqq M^{\prime}|x-y| .
$$

In other words, $Q(x, u)$ satisfies precisely the hypotheses of Lemma 7, with $S$ replaced by $S_{0}$.

The next step is to integrate (37) over $S_{0}$. Before writing down the result of this integration, it is convenient to estimate the integral of the right hand side. Specifically, since $u_{m} \rightarrow u$, one has essentially by Lebesgue's bounded convergence theorem

$$
\begin{gathered}
\int_{s_{0}}\left[g\left(x, u_{m}, p_{h}\right)-g\left(x, u, p_{h}\right)\right] d x \rightarrow 0, \\
\int_{S_{0}} p_{h} \cdot\left[g_{p}\left(x, u_{m}, p_{h}\right)-g_{p}\left(x, u, p_{h}\right)\right] d x \rightarrow 0,
\end{gathered}
$$

as $m \rightarrow \infty$. The remaining difference between terms on the second and third lines of (37) can be estimated by Lemma 7. Hence, making use of (36) we obtain

$$
I_{o}\left[u_{m}, S_{0}\right]-I_{o}\left[u, S_{0}\right] \geqq-n M^{\prime} \int_{S_{0}}\left|u_{m}-u\right| d x-\epsilon_{1}-2 M \epsilon,
$$

where $\epsilon_{1}$ tends to zero as $m \rightarrow \infty$. Now letting $m \rightarrow \infty$ through an appropriate subsequence yields

$$
\lim \inf I_{\theta}\left[u_{m}, S_{0}\right]-I_{\theta}\left[u, S_{0}\right] \geqq-2 M \epsilon .
$$


Since $\epsilon$ was arbitrary and since $g$ vanishes outside $S_{0}$, this implies $I_{\theta}[u, R]$ $\leqq \lim$ inf $I_{0}\left[u_{m}, R_{m}\right]$, completing the proof.

The above argument is a generalization and extension of Tonelli's classical proof of the lower semicontinuity of variational integrals [18].

12. Proof of Theorem 13. This result can be obtained by essentially the same steps which were used to prove Theorem 12. In particular, we observe that Lemma 8 holds for an arbitrary integrand $f$ provided the first condition is replaced by

$$
g \leqq f+\epsilon(1+|p|) .
$$

In addition, we recall from the preceding section that lower semicontinuity holds for any integrand $g$ satisfying conditions (iii) and (iv) of Lemma 8 .

As in the proof of Theorem 12, therefore, let $f$ be an arbitrary integrand, let $S$ be a compact subset of $R$, and let $\epsilon$ be a small positive number. If $g$ denotes the integrand associated with $f, S$, and $\epsilon$ as above, we have

$$
\begin{aligned}
I_{o}[u, S] & \leqq \lim \inf I_{o}\left[u_{m}, R_{m}\right] \\
& \leqq \lim \inf I\left[u_{m}, R_{m}\right]+\epsilon\left(\text { Meas } S_{0}+\lim \sup D\left[u_{m}, R_{m}\right]\right) .
\end{aligned}
$$

By hypothesis the last term on the right is no larger than $H$. Thus letting $\epsilon \rightarrow 0$ and then $S \rightarrow R$ yields the required inequality.

Theorem 13 can be given an apparently more general form if we make use of Rellich's lemma. In particular, we may replace local convergence in Theorem 13 by weak local convergence, or even convergence in measure. The reason for this is that any family of functions for which $D[u]$ is uniformly bounded, and such that $|u|$ is uniformly bounded on sets of positive measure, is locally compact in $L_{p}$ for any $p<n /(n-1)$. Thus from any sequence $\left\{u_{m}\right\}$ which converges either weakly or in measure to a function $u$, we can extract a subsequence $\left\{u_{m^{\prime}}\right\}$ which converges locally to $u$. Thus, neither weak convergence nor convergence in measure can possibly lead to a smaller value of lim inf $I\left[u_{m}, R_{m}\right]$ than $I[u, R]$, proving our assertion.

We remark also that Theorem 13 is slightly stronger than the related Theorem 4.1 of [8]. To see this, observe that the latter theorem divides naturally into two distinct parts; first, lower semicontinuity for non-negative convex integrands, and second, continuity of integrals of the form

$$
\mathscr{L}[u]=\int_{S}\left[a(x, u) \cdot u_{x}+b(x, u) u+c(x, u)\right] d x,
$$

where $a, b, c$ are uniformly bounded continuous functions. Morrey requires for both parts that $\left\{u_{m}\right\}$ and $\left\{u_{m x}\right\}$ should converge weakly in $L_{1}$ to $u$ and $u_{x}$, respectively. As regards the continuity of $\mathcal{L}[u]$ this type of convergence is certainly necessary. On the other hand, for non-negative convex integrands this convergence is stronger than needed: according to Theorem 13 and the remarks of the preceding paragraph it is enough that $\left\{u_{m}\right\}$ should converge 
weakly to $u$ and that the integrals $D\left[u_{m}\right]$ should be uniformly bounded. (It may be added that this latter kind of convergence is also sufficient to insure the continuity of $\mathscr{L}[u]$ provided that $a$ has compact support in $S$ and is uniformly continuous in $x$, whatever the value of $u$.)

13. Concluding remarks. In this section we shall discuss some extensions of the previous work, and also certain alternate definitions of the functional $\boldsymbol{g}[u]$.

Vectorfunctions. The case of vector-valued argument functions $u=$ $\left(u^{1}, \cdots, u^{\nu}\right)$ is of considerable interest, and to some extent allows treatment by our present methods. For vector functions $u$ the integrand depends on the set of partial derivatives $\partial u^{i} / \partial x_{\alpha}, i=1, \cdots, \nu ; \alpha=1, \cdots, n$, and we write correspondingly $f=f(p)=f\left(p_{\alpha}^{i}\right)$. We say that $f$ is quasi-convex in $p$ if the function

$$
f\left(p_{\alpha}^{i}+\lambda \xi_{\alpha} \eta^{i}\right)
$$

is convex in $\lambda$ for all fixed values of the vectors $p, \xi$, and $\eta$. It is known that quasi-convexity is essentially a necessary and sufficient condition that an extremal furnish a weak relative minimum to the integral $I[u]$. In this sense quasi-convexity is the appropriate generalization to vector problems of the convexity condition for scalar problems. We also observe that for $\nu>1$ convexity implies quasi-convexity, while for $\nu=1$ the two concepts are equivalent.

The results of Part I and II can easily be extended to the important subclass of quasi-convex integrands which satisfy the condition

$$
f \geqq 0, \quad f \text { convex in } p \text {. }
$$

In fact only minor changes in the proofs are necessary in order to see that Theorems 1 through 11 remain true in this case. Theorems 12 and 13, however, no longer hold for vector functions even under the condition $\left(8^{\prime}\right)$, though by analyzing the proofs the reader will no doubt be able to discover various additional conditions which will make these results valid.

For the general case of quasi-convex integrands the writer has been unable to obtain any comparable results, and apparently some new ideas will be necessary $\left({ }^{(4)}\right.$.

Other definitions of $\mathscr{g}[u]$. Certain slightly different definitions of $\mathscr{g}[u]$ might be proposed besides the one given in $\$ 1$. We could, for example, consider alternate types of convergence of $\left\{u_{m}\right\}$ to $u$, or even different classes of functions $u_{m}$. As regards alternate modes of convergence, the most important possibilities are:

(a) Convergence almost everywhere, or more generally, convergence in measure.

(ฯ) See, however, C. B. Morrey, Jr., Pacific J. Math. vol. 2 (1952). 
(b) Weak convergence, as in $\$ 5$.

(c) Uniform convergence.

(d) Convergence on all of $R$ rather than local convergence, with $g[u, R]$ $=$ Minimum limit $I\left[u_{m}, R\right]$.

To discuss these possibilities, we observe first that if the integrand is such that $D[u]$ is always less than a fixed multiple of $\mathscr{g}[u]$, then by Rellich's lemma both (a) and (b) are equivalent to local convergence. Therefore in this case, at any rate, there is no real gain of generality in admitting weak convergence or convergence in measure. On the other hand, if $D[u]$ is not majorized by a multiple of $g[u]$, then I do not see how to construct a reasonable theory for convergence in measure unless at the same time $u$ is required to be continuous, nor for weak convergence unless the integrand is of type I and independent of $u$.

In case (c) we are led immediately to continuous functions $u$, so that the theory is less general than the present one.

Case (d) is more interesting. Letting $g^{*}[u]$ denote the functional which arises in this case (and reserving the notation $g[u]$ for the functional defined in $\$ 1$ ), we have generally

$$
g[u] \leqq g^{*}[u] .
$$

Also, if $f$ satisfies the hypotheses of Lemma 6, if $R$ is bounded, and if $u$ is strongly differentiable in some larger set, then

$$
g^{*}[u] \leqq I[u] .
$$

Relations (38) and (39) can be used together with our earlier results to derive various theorems connecting $I, \mathfrak{g}$, and $\mathfrak{g}^{*}$. We mention just one example:

Suppose that $f=f(p)$. Then for every weakly differentiable function we have $I[u] \leqq g[u] \leqq g^{*}[u]$. If $R$ is bounded and $u$ is strongly differentiable on some larger set, then $I[u]=g[u]=g^{*}[u]$.

It seems that beyond the class of strongly differentiable functions one usually has $g[u]<g^{*}[u]$. In at least one important case, however, the functionals $\mathcal{g}$ and $\mathscr{g}^{*}$ are identical. This occurs in particular when

$$
\left\{\begin{array}{l}
f \leqq A(1+|p|), \text { and } f \text { is of type I or type II, } \\
u \text { is uniformly continuous in } R, \\
R \text { has a smooth boundary. }
\end{array}\right.
$$

A proof that $g \equiv g^{*}$ in this case can be given along the lines indicated in [15, $\S 5$. ] If there are only two independent variables one can replace the condition that $R$ has a smooth boundary by the conditon that $R$ has a rectifiable boundary.

Turning next to the possibility of using other than continuously differentiable functions $u_{m}$ in the definition of $g[u]$, natural alternatives which suggest themselves are quasi-linear functions, Lipschitz functions, and strongly differentiable functions. The first two of these lead to exactly the functional $g[u]$, and we need not consider them further. The functional $\boldsymbol{g}_{\boldsymbol{s}}[u]$ which re- 
sults from using strongly differentiable functions need not be identical with $g[u]$. The two functionals agree for integrands satisfying the hypotheses of Lemma 6 , but beyond this we generally have $g_{S}[u] \leqq g[u]$.

The theory of the functional $\Im_{S}[u]$ is in some ways more elegant than that of $\mathscr{g}[u]$. For example, since $\mathscr{g}_{S}[u] \leqq I[u]$ for any strongly differentiable function, it is evident that the conclusion of Theorem 9 could be somewhat strengthened; moreover if $I[u]$ is lower semicontinuous (as in Theorems 11 and 12), then $\mathscr{g}_{S}[u]=I[u]$ for strongly differentiable functions. Of course, the functional $\boldsymbol{g}_{\boldsymbol{S}}$ is not as elementary as $\boldsymbol{g}$, so that the gain in elegance is paid for in another way.

Note. The above research was supported in part by the United States Air Force Office of Scientific Research under Contract No. 49(638)-262.

\section{REFERENCES}

1. S. Cinquini, Condizioni sufficienti per la semicontinuitd nel calcolo delle variazioni, Ann. Scuola Norm. Sup. Pisa (2) vol. 2 (1933) pp. 41-58.

2. L. Giuliano, Sulle condizioni sufficienti per la semicontinuitd degli integrali doppi del calcolo delle variazioni, Ann. Scuola Norm. Sup. Pisa (2) vol. 10 (1941) pp. 37-56.

3. C. Goffman, Lower semicontinuity and area functionals, Rend. Circ. Mat. Palermo (2) vol. 2 (1953) pp. 203-235.

4. P. Halmos, Measure theory, New York, Van Nostrand, 1950.

5. K. Krickeberg, Distributionen, Funktionen beschränkter Variation, und Lebesguescher Inhalt nichtparametricher Flächen, Ann. Mat. Pura. Appl. vol. 44 (1957) pp. 105-133.

6. E. J. McShane, On a certain inequality of Steiner, Ann. of Math. vol. 33 (1932) pp. 125-138.

7. E. J. McShane and T. A. Botts, Real analysis, Princeton, Van Nostrand, 1959.

8. C. B. Morrey, Multiple integral problems in the calculus of variations and related topics, Univ. California Publ. Math., 1943. Especially Chapter III.

9. M. Nagumo, Über die gleichmässige Summierbarkeit und ihre Anwendung auf ein Variationsproblem, Jap. J. Math. vol. 6 (1929) pp. 173-182. See also L. M. Graves, The theory of functions of real variables, New York, McGraw-Hill, 1946, p. 235.

10. C. Y. Pauc, La methode métrique en calcul des variations, Paris, Hermann, 1941. Especially p. 54 .

11. S. Saks, Theory of the integral, 2d ed., Hafner, 1937.

12. L. Schwartz, Theorie des distributions, II, Paris, Hermann, 1951.

13. J. Serrin, $A$ new definition of the integral for non-parametric problems in the calculus of variations, Acta Math. vol. 102 (1959) pp. 23-32.

14. - On the differentiability of functions of several variables, Arch. Rational Mech. Anal. vol. 7 (1961) pp. 359-372. $435-440$.

15. - On the area of a surface $z=f(x, y)$, Amer. Math. Monthly vol. 68 (1961) pp.

16. A. G. Sigalov, Two dimensional problems of the calculus of variations in non-parametric form, Trudy Moskov. Mat. Obšx. vol. 2 (1953) pp. 201-233.

17. L. Tonelli, Sulla quadratura delle superficie, Rend. Accad. Naz. Lincei (6) vol. 3 (1926) pp. 357-363, 445-450, 633-638.

18. - Sur la semi-continuité des integrales doubles du calcul des variations, Acta Math., vol. 53 (1929) pp. 325-346. 11-32.

19. I. Verchenko, Über das Flächenmass von Mengen, Mat. Sb. (N.S.) vol. 10 (1942) pp.

University of MinNesota, Institute of Technology, MinNEAPOLIS, MinNESOTA 